\title{
Morphology-induced redistribution of surface plasmon modes in two-dimensional crystalline gold platelets
}

S. Viarbitskaya, A. Teulle, A. Cuche, J. Sharma, C. Girard, E. Dujardin, and A. Arbouet

Citation: Appl. Phys. Lett. 103, 131112 (2013); doi: 10.1063/1.4823533

View online: https://doi.org/10.1063/1.4823533

View Table of Contents: http://aip.scitation.org/toc/apl/103/13

Published by the American Institute of Physics

\section{Articles you may be interested in}

Interaction of an ultrashort optical pulse with a metallic nanotip: A Green dyadic approach

Journal of Applied Physics 112, 053103 (2012); 10.1063/1.4747840

Near-field optical properties of single plasmonic nanowires

Applied Physics Letters 89, 233119 (2006); 10.1063/1.2403914

Near-field optical imaging of plasmon modes in gold nanorods

The Journal of Chemical Physics 122, 154701 (2005); 10.1063/1.1873692

Multipolar excitation in triangular nanoprisms

The Journal of Chemical Physics 123, 114713 (2005); 10.1063/1.2046633

Sculpting nanometer-sized light landscape with plasmonic nanocolumns

The Journal of Chemical Physics 131, 224707 (2009); 10.1063/1.3271794

Spectroscopy of single metallic nanoparticles using total internal reflection microscopy

Applied Physics Letters 77, 2949 (2000); 10.1063/1.1323553

\section{PHYSICS TODAY}

MANAGER'S GUIDE

WHITEPAPERS

\section{READ NOW}

PRESENTED BY

Accelerate R\&D with
Multiphysics Simulation 


\title{
Morphology-induced redistribution of surface plasmon modes in two-dimensional crystalline gold platelets
}

\author{
S. Viarbitskaya, A. Teulle, A. Cuche, J. Sharma, C. Girard, E. Dujardin, ${ }^{\text {a) }}$ and A. Arbouet ${ }^{\text {b) }}$ \\ CEMES, CNRS UPR 8011, University of Toulouse, 29 rue Jeanne Marvig, BP 94347, 31055 Toulouse, France
}

(Received 22 July 2013; accepted 12 September 2013; published online 27 September 2013)

\begin{abstract}
The 2D optical field intensity distribution in sub-micron, ultrathin, and crystalline gold platelets is investigated by two-photon luminescence (TPL) microscopy. In particular, the evolution of the TPL maps as the particle morphology undergoes a transition from triangular to hexagonal reveals that the signatures of the high-order surface plasmon states sustained by the platelets follows the same $\mathrm{C}_{3 v}$ to $\mathrm{C}_{6 v}$ symmetry redistribution. Experimental observations are precisely accounted for by theoretical simulations based on the Green dyadic method. (C) 2013 AIP Publishing LLC.

[http://dx.doi.org/10.1063/1.4823533]
\end{abstract}

Metallic nanostructures possess unique optical properties originating from the existence of Surface Plasmon Polaritons (SPP).${ }^{1-3}$ These hybrid optical excitations, which couple electron density waves with the electromagnetic field at a metal-dielectric interface, allow the enhancement, the confinement and, ultimately, the manipulation of light at the nanoscale. ${ }^{4,5}$ These possibilities have boosted the emergence of plasmonics and its application in integrated opto-electronics, ${ }^{6}$ information processing, ${ }^{7}$ sensing, ${ }^{8,9}$ labeling, ${ }^{10}$ and nanomanipulation. ${ }^{11}$ The design of devices based on plasmonic nanostructures as elementary building blocks requires efficient strategies to control the spatial distribution and spectral features of SPP modes. From a general point of view, confining a wave in some finite region yields modes that depend both upon the constituting material and the boundary conditions. For instance, engineering nanostructures from individual atoms or molecules confines twodimensional surface electronic states into closed resonators or "quantum corrals," which are directly imaged using a scanning tunneling microscope. ${ }^{12}$ An optical analogue was demonstrated with surface photonic states confined inside optical corrals made of gold pads on a transparent substrate. ${ }^{13,14}$ The electron density waves associated with surface plasmons (SP) can be tailored in a similar fashion through a careful design of the supporting metallic nanostructure. Depending upon the particle morphology, a variety of SPP modes have been obtained such as Fabry-Perot ${ }^{15}$ or whispering gallery modes ${ }^{16}$ for instance. These investigations have demonstrated that colloidal chemistry is better performing than standard lithography techniques for the fabrication of single crystalline metallic nanostructures: the reduced dissipation due to intrinsic losses in the material and the sharp geometries defined by the crystallographic facets favours the build-up of well-defined SP modes. ${ }^{15}$

In this context, it has recently been shown that colloidal gold platelets sustain high-order delocalized surface plasmon modes, arising from the two-dimensional (2D) confinement of the electron oscillation. ${ }^{17-19}$ Indeed, multiple interferences between delocalized SPPs yield optical near-fields that are

\footnotetext{
a) erik.dujardin@cemes.fr

b)arnaud.arbouet@cemes.fr
}

strongly localized at specific hot spots along the edges of the platelets. ${ }^{17}$ These investigations suggest that the control of the spatial and spectral properties of the SP modes and the related SP density of states (SP-DOS) of two-dimensional metallic nanostructures could provide a tool for designing plasmonic devices for optical information processing at the nanoscale. ${ }^{19}$ In this letter, we use two-photon luminescence (TPL) microscopy to study the influence of the particle morphology on the SP modes of planar plasmonic nanostructures prepared by an original bottom-up nanofabrication technique. ${ }^{19}$ The spatial distribution of the plasmon modes is monitored and compared to numerical simulations based on the Green dyadic method as the particle in-plane shape undergoes a symmetry transition, from triangular $\left(\mathrm{C}_{3 v}\right)$ to hexagonal shape $\left(\mathrm{C}_{6 v}\right)$. In addition, for a given geometry, TPL maps are recorded for different combinations of wavelength, incident light polarization, and nanostructure size.

Suspensions of 20-nm thick gold triangular, truncated triangular, and hexagonal nanoprisms of typical lateral size ranging from 0.5 to $1.0 \mu \mathrm{m}$ were prepared using a one-pot protocol based on the direct reduction of Au precursors by polyvinylpyrrolidone (PVP) in alkaline conditions at room temperature. A small volume of the particle suspension was drop-casted onto $150 \mu \mathrm{m}$-thick, thoroughly cleaned glass substrates coated with $10-20 \mathrm{~nm}$ of conductive indium tin oxide. SEM imaging (Zeiss 1540XB) was performed in order to identify individual prisms and measure their lateral dimensions. SEM was systematically performed after all optical spectroscopy experiments to ascertain that the particles retained their morphology. Figure 1 shows the absorption spectrum of the colloidal suspension of gold nanoplatelets. A broad feature with a peak at $1450 \mathrm{~nm}$ is ascribed to the dipolar resonance of the platelets. A detailed examination of the $500-1200 \mathrm{~nm}$ range reveals a number of smaller features that were further investigated by dark field (DF) microscopy on individual particles (inset of Fig. 1). DF spectra evidence several optical resonances lying at higher energies, which are assigned to higher order plasmon modes analogous to the Fabry-Perot cavity modes of metallic nanowires. ${ }^{15,17}$ The dramatic influence of the particle morphology on these SP modes can be noticed if one compares the far-field optical spectra measured either on particles with different shape 
((ii) vs (iii)) or particles with similar in-plane shape but different sizes ((iii) vs (iv)).

Furthermore, we have performed TPL microscopy experiments and numerical simulations to investigate the influence of the particle morphology on the optical near-field intensity distribution of the SP modes detected by dark-field spectroscopy in the near-infrared range (Fig. 1). Two-photon excited photoluminescence is an inefficient light emission process in the case of smooth metallic films, but it can be strongly enhanced whenever SP resonances are excited in metallic nanostructures. ${ }^{20}$ Its intensity is proportional to the fourth power of the local electric field which makes TPL microscopy a very attractive technique for high resolution optical field intensity mapping in plasmonic nanostructures. ${ }^{21-24}$ Recently, we have shown that the TPL signal can be equivalently described as the convolution of the SP-DOS in the metallic nano-object with the intensity spatial profile of the optical excitation. ${ }^{19}$ The sample is mounted on a XY piezostage and raster scanned with a femtosecond laser beam $(80 \mathrm{MHz}, 120 \mathrm{fs}, 700-800 \mathrm{~nm})$, tightly focused through a high numerical aperture oil immersion microscope objective (Olympus $100 \times$, NA 1.35). The polarization of the incident light is controlled with a $\lambda / 2$ plate. The laser power is kept in the $100-300 \mu \mathrm{W}$ range at the back aperture of the microscope objective. TPL intensity is recorded in epi-collection geometry using a dichroic mirror, filtered, and focused on a photomultiplier tube (PMT) operated in analog mode. More details about our experimental setup are given in Ref. 19. The excitation wavelength has been chosen in the $700-800 \mathrm{~nm}$ spectral range in which high-order SP resonances of the gold nanoplatelets can be excited (Fig. 1). We have first investigated the TPL signal on three particles with different in-plane shapes, the SEM images of which are shown in Figures 2(a)-2(c). The first two particles, triangular and truncated triangular, possess a $C_{3 v}$ in-plane symmetry whereas the third hexagonal one has a $C_{6 v}$ symmetry. All three particles have the same thickness of $20 \mathrm{~nm}$. Figures 2(d)-2(i) show the TPL maps detected on these particles. Triangular nanoprisms yield one or two intense TPL spots depending on the polarization direction of the optical

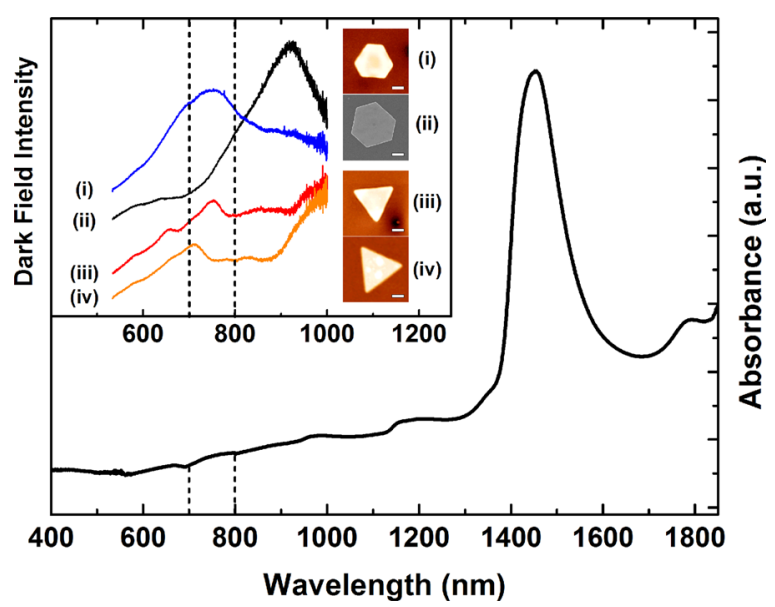

FIG. 1. Absorption spectrum measured on a suspension of gold nanoplatelets. Inset: dark-field spectra measured on individual nanoparticles deposited on a glass substrate together with the corresponding AFM ((i), (iii), and (iv)) or SEM (ii) image. The dashed lines delimit the spectral range of the TPL excitation wavelength. Scale bars are $200 \mathrm{~nm}$. excitation (Figs. 2(d) and 2(g)). The sequential switching between one (Fig. 2(d)) and two-spot patterns (Fig. 2(g)) in triangular platelets obtained with a rotating polarization has been thoroughly described in Ref. 19. Truncated triangular nanoprisms produce TPL maps featuring three intense spots situated at the truncations with a relative intensity depending on the incident light polarization (Figs. 2(e) and 2(h)). However, the truncation of the corners leads to a more homogeneous TPL intensity distribution with respect to the perfectly triangular case. The overall pattern still follows the $C_{3 v}$ symmetry. The comparison of Figures $2(\mathrm{~g})$ and $2(\mathrm{~h})$ makes it clear that the elongated TPL pattern obtained at the bottom of the truncated platelet for a vertical polarization arises from the merging of the two TPL spots located at the apices in Figure 2(g). This evolution of the TPL intensity patterns is even more obvious when considering a hexagonal particle. In Figure 2(i), the intense signal in the bottom region is further shrunk into a single bright oblong lobe while the small spot on the upper edge is reinforced. Such a morphology-induced redistribution of the TPL intensity also occurs for the horizontal polarization. The bright lobe visible on the sharp triangle (Fig. 2(d) gradually disappears with increasing truncation (Figs. 2(e) and 2(f)) as other features appear on the left and right sides of the truncated triangle and hexagon. Noteworthy, in all our experiments, the measured TPL vanishes in the center of the particles. These observations are in good agreement with the Electron Energy Loss Spectroscopy experiments reported by Gu et al. ${ }^{17}$

To analyse our results, we have performed numerical simulations using the three-dimensional Green dyadic method, which is particularly well-suited for particles of complex geometries deposited on a substrate. ${ }^{25}$ To closely account for our experimental configuration, we have combined a realistic description of the focused optical excitation and a generalized field propagator to simulate the experimental TPL maps. Further details on our method are given in Ref. 26. The TPL signal is computed from the local electric field distribution $\mathbf{E}\left(\mathbf{R}_{0}, \mathbf{r}, \omega\right)$ generated inside the platelets, where $\mathbf{R}_{0}$ is the position of the light beam center and $\omega$ is the angular frequency of the laser

$$
\mathcal{I}_{T P L}\left(\mathbf{R}_{0}, \omega\right) \propto \int_{V}\left[\left|\mathbf{E}\left(\mathbf{R}_{0}, \mathbf{r}, \omega\right)\right|^{2}\right]^{2} d \mathbf{r} .
$$

In the latter expression, the integration is performed on the volume $V$ of the metallic nanostructure. The simulated TPL maps shown in panels (j)-(o) of Figure 2 are in excellent agreement with our experiments: both the overall distribution of the TPL signal and the relative intensities of the spots are very well reproduced. Residual discrepancies could be explained by uncertainties in particle orientation with respect to laser polarization. Both experimental and theoretical data show that the strong localization of the TPL signal at the apices is a characteristic feature of the sharp prisms and suggest that acute corners play a specific role in the generation of strong local electric fields (Figs. 2(d) and 2(g)). The redistribution of the optical field intensity evidenced in Figure 2 as the platelet in-plane shape gradually changes from perfectly triangular to hexagonal illustrates the strong sensitivity of the optical field intensity distribution to boundary conditions 


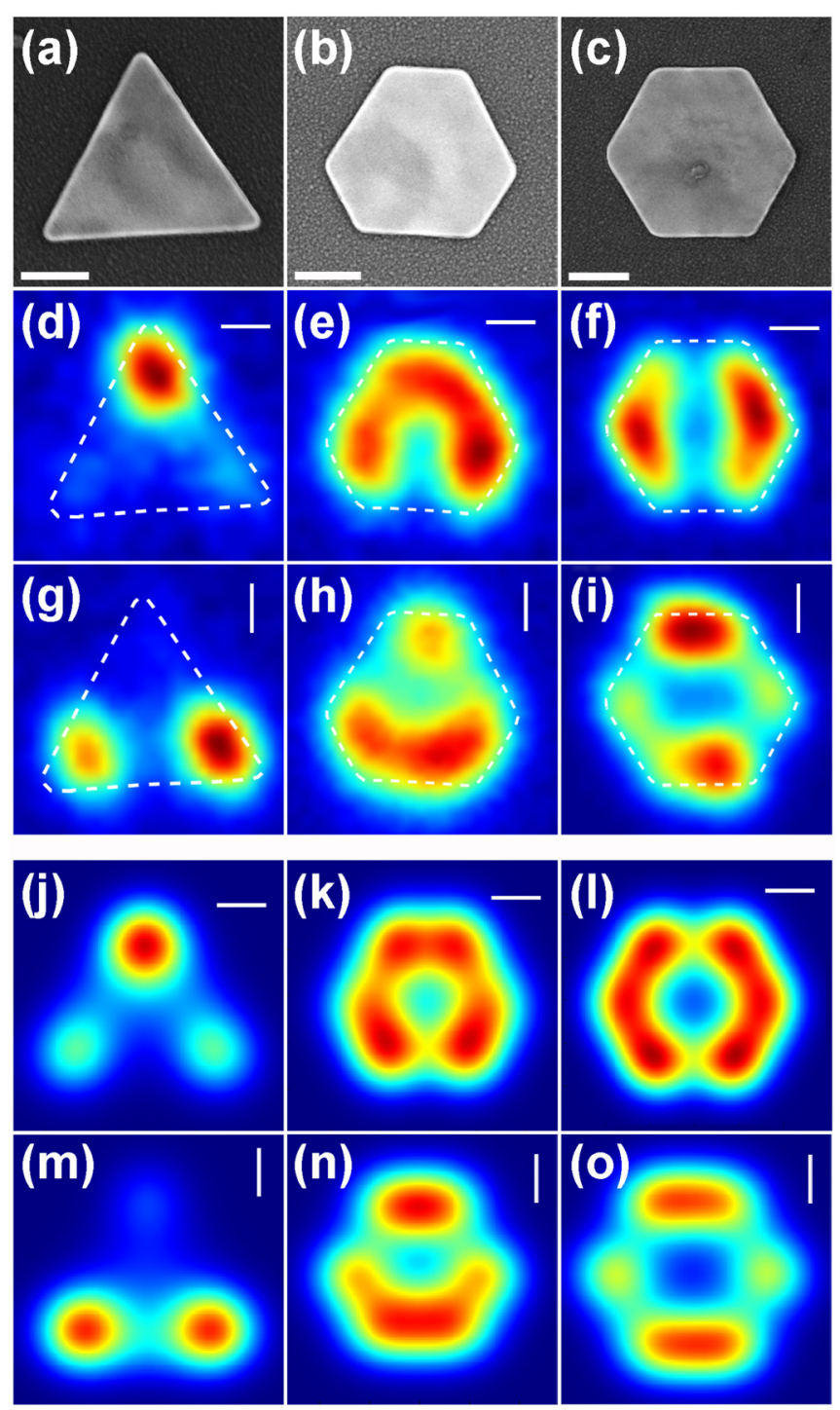

FIG. 2. (a)-(c) SEM images of triangular, truncated triangular, and hexagonal gold nanoplatelets. (d)-(i) Corresponding TPL maps acquired with $\lambda=700 \mathrm{~nm}$ and linear polarization of $0^{\circ}(\mathrm{d})-(\mathrm{f})$ and $90^{\circ}(\mathrm{g})-(\mathrm{i})$, as indicated by the white bars in the upper right corners. (j)-(o) Corresponding simulated maps. Scale bars are $200 \mathrm{~nm}$.

(number, length, and relative orientation of edges). Consistently, one can anticipate that a homothetic size variation should also induce a spatial redistribution of the TPL pattern. Figures 3(c)-3(f) show TPL maps obtained with $800 \mathrm{~nm}$ excitation on two hexagonal platelets of different sizes and for two orthogonal polarizations. The perimeters of these particles displayed in Figures 3(a) and 3(b) are $1920 \mathrm{~nm}(320 \mathrm{~nm}$ edge length) and $2820 \mathrm{~nm}$ (approximately $470 \mathrm{~nm}$ edge length) respectively. The smaller hexagon exhibits two broad areas yielding intense TPL aligned perpendicularly to the polarization direction (Figs. 3(c) and 3(e)). On the contrary, Figures 3(d) and 3(f) show that the main TPL patterns on the larger hexagonal prism at the same excitation wavelength are aligned along the polarization direction. It thus appears that the two-lobe pattern can be flipped by either rotating the incident polarization (for example Figs. 3(c) and 3(e)) or by increasing the particle size (for example Figs. 3(c) and 3(d)). These observations, together with the dark-field spectroscopy experiments presented in Figure 1, suggest that the observed intensity patterns are the signatures of different SP modes. Interestingly, the larger hexagon allows to resolve smaller features that distinguish the two symmetry cases where the polarization links two parallel edges (Fig. 3(d)) or two opposite apices (Fig. 3(f)). In the former case, the full pattern is composed of two straight oblong and two small circular spots while in the latter only two V-shaped features are observed. Although it is difficult to resolve all features in the smaller hexagon, a similar trend can be inferred from the images. A closer examination of the simulated images (Figs. 2(k) and 2(1), 2(n) and 2(o)) clarifies the truncation-induced evolution of the TPL patterns. Indeed, patterns in the truncated triangle and hexagon can be constructed from six luminescent areas located at the six apex. The incident polarization modulates the relative intensities of each area, which merge together when intense and according to their mutual proximity imposed by the truncation. Thus in Figure 2(k), one observes

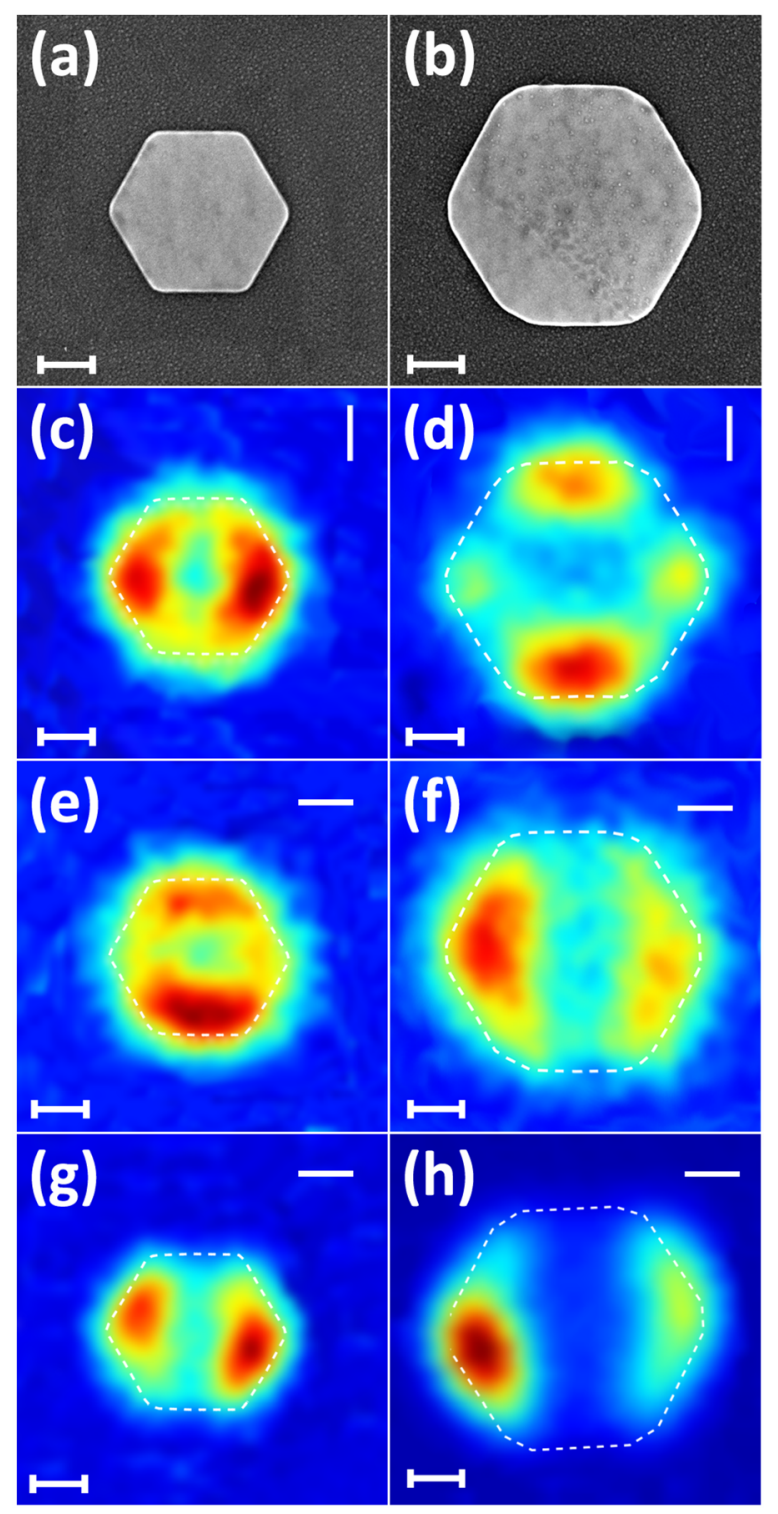

FIG. 3. (a), (b) SEM images of the hexagonal prisms with perimeters of $1910 \mathrm{~nm}$ (320 nm edge length) and $2820 \mathrm{~nm}$ (approximately $470 \mathrm{~nm}$ edge length, slightly truncated), respectively. (c)-(f) Corresponding TPL maps acquired with $800 \mathrm{~nm}$ linearly polarized light, as indicated by the white bars in the upper right corners. (g), (h) TPL map was acquired with $700 \mathrm{~nm}$ excitation. Scale bars are $200 \mathrm{~nm}$. 
essentially three pairs of merged spots along each truncation, while in Figures 2(l) and 3(f) the spots on the hexagon apex are grouped by three, following the symmetry imposed by the horizontal polarization. This also accounts for the large area of four and opposite pair of merged spots in Figure 2(n) as well as the two pairs of merged spots and two lone features in Figures 2(o) and 3(d).

In a recent study, we have shown that the TPL intensity distribution measured on triangular prisms changes dramatically when excitation wavelength was increased from 700 to $850 \mathrm{~nm} .{ }^{19}$ This observation was attributed to the excitation of different SP modes in the triangular platelets. To support this interpretation, we have performed the same experiment as in Figures 3(e) and 3(f) but for a shorter excitation wavelength $(700 \mathrm{~nm})$. Figure $3(\mathrm{~g})$ shows that this change in wavelength also results in a $90^{\circ}$ rotation of the TPL pattern relative to that obtained with $800 \mathrm{~nm}$ excitation (Fig. 3(e)). This observation suggests that in the case of the small prism, the modification of the excitation wavelength allows addressing a different SP mode. It should be emphasized that Figure 3(g) is similar to Figure 3(f) but with both smaller excitation wavelength and structure size. This scaling effect, similar to what has been extensively investigated on optical antennas, is a consequence of the excitation of SP modes delocalized on the platelets. However, it is important to note that the change in TPL pattern is not observed in all cases. Figure 3(h) shows that in the case of the larger prism, the decrease in excitation wavelength from $800 \mathrm{~nm}$ to $700 \mathrm{~nm}$ is not sufficient to excite a different SP mode and modify notably the TPL map.

In summary, two-photon photoluminescence imaging and numerical simulations were used to investigate the distribution of the optical field intensity in submicronic thin gold platelets. The planar morphology and single crystallinity of these nanosystems allows the build-up of delocalized SP modes that produce subwavelength intensity patterns defined solely by the particle morphology and excitation wavelength. Our results paves the way to the engineering of the spectral and spatial characteristics of surface plasmon modes through the control of the morphology of single crystalline gold platelets and their subsequent use in future planar optical devices. ${ }^{19}$

The authors acknowledge financial support from the European Research Council (ERC, Contract No. ERC
2007-StG Nr 203872 COMOSYEL), Agence Nationale de la Recherche (ANR, grant ANR-09-BLAN-0049-01 Plastips), the CPER Gaston Dupouy 2007-2013 and the massively parallel computing centre CALMIP in Toulouse.

${ }^{1}$ W. L. Barnes, A. Dereux, and T. W. Ebbesen, Nature 424, 824 (2003).

${ }^{2}$ L. Novotny and B. Hecht, Principles of Nano-Optics (Cambridge University Press, New York, 2006).

${ }^{3}$ N. J. Halas, S. Lal, W.-S. Chang, S. Link, and P. Nordlander, Chem. Rev. 111, 3913 (2011).

${ }^{4}$ E. Ozbay, Science 311, 189 (2006).

${ }^{5}$ J. A. Schuller, E. S. Barnard, W. Cai, Y. C. Jun, J. S. White, and M. L. Brongersma, Nat. Mater. 9, 193 (2010).

${ }^{6}$ S. A. Maier and H. A. Atwater, J. Appl. Phys. 98, 011101 (2005).

${ }^{7}$ Z. Jacob and V. M. Shalaev, Science 334, 463 (2011).

${ }^{8}$ A. Kinkhabwala, Z. F. Yu, S. H. Fan, Y. Avlasevich, K. Mullen, and W. E. Moerner, Nat. Photonics 3, 654 (2009).

${ }^{9}$ T. Schumacher, K. Kratzer, D. Molnar, M. Hentschel, H. Giessen, and M. Lippitz, Nat. Commun. 2, 333 (2011).

${ }^{10}$ J. L. Li and M. Gu, Biomaterials 31, 9492 (2010).

${ }^{11}$ M. Righini, G. Volpe, C. Girard, D. Petrov, and R. Quidant, Phys. Rev. Lett. 100, 186804 (2008).

${ }^{12}$ E. J. Heller, M. F. Crommie, C. P. Lutz, and D. M. Eigler, Nature 369, 464 (1994).

${ }^{13}$ G. Colas des Francs, C. Girard, J.-C. Weeber, C. Chicane, T. David, A. Dereux, and D. Peyrade, Phys. Rev. Lett. 86, 4950 (2001).

${ }^{14}$ C. Chicanne, T. David, R. Quidant, J. C. Weeber, Y. Lacroute, E. Bourillot, A. Dereux, G. Colas des Francs, and C. Girard, Phys. Rev. Lett. 88, 097402 (2002).

${ }^{15}$ H. Ditlbacher, A. Hohenau, D. Wagner, U. Kreibig, M. Rogers, F. Hofer, F. R. Aussenegg, and J. R. Krenn, Phys. Rev. Lett. 95, 257403 (2005).

${ }^{16}$ B. Min, E. Ostby, V. Sorger, E. Ulin-Avila, L. Yang, X. Zhang, and K. Vahala, Nature 457, 455 (2009).

${ }^{17}$ L. Gu, W. Sigle, C. T. Koch, B. gt, P. A. van Aken, N. Talebi, R. Vogelgesang, J. Mu, X. Wen, and J. Mao, Phys. Rev. B 83, 195433 (2011).

${ }^{18}$ T. A. Major, M. S. Devadas, S. S. Lo, and G. V. Hartland, J. Phys. Chem. C 117, 1447 (2013).

${ }^{19}$ S. Viarbitskaya, A. Teulle, R. Marty, J. Sharma, C. Girard, A. Arbouet, and E. Dujardin, Nat. Mater. 12, 426 (2013).

${ }^{20}$ A. Bouhelier, R. Bachelot, G. Lerondel, S. Kostcheev, P. Royer, and G. P. Wiederrecht, Phys. Rev. Lett. 95, 267405 (2005).

${ }^{21}$ M. R. Beversluis, A. Bouhelier, and L. Novotny, Phys. Rev. B 68, 115433 (2003).

${ }^{22}$ P. J. Schuck, D. P. Fromm, A. Sundaramurthy, G. S. Kino, and W. E. Moerner, Phys. Rev. Lett. 94, 017402 (2005).

${ }^{23}$ P. Ghenuche, S. Cherukulappurath, T. H. Taminiau, N. F. van Hulst, and R. Quidant, Phys. Rev. Lett. 101, 116805 (2008).

${ }^{24}$ H. Okamoto and K. Imura, Prog. Surf. Sci. 84, 199 (2009).

${ }^{25}$ C. Girard, Rep. Prog. Phys. 68, 1883 (2005).

${ }^{26}$ A. Teulle, R. Marty, S. Viarbitskaya, A. Arbouet, E. Dujardin, C. Girard, and G. Colas des Francs, J. Opt. Soc. Am. B 29, 2431 (2012). 